\title{
Patellofemoral joint preservation
}

\author{
John P. Fulkerson
}

Published online: 4 November 2008

(C) Springer-Verlag 2008

The article by Gigante et al. documents what we have seen in my practice for over 20 years, and I congratulate them on their careful documentation and pursuit of this logical approach to patellofemoral joint preservation. This is what is so pertinent about their work: preservation versus replacement of the degenerated patellofemoral joint, particularly when the degeneration is related to PF joint malalignment. In younger patients, preservation should always be the first choice. Carofino has recently documented in the Journal of Knee Surgery that anteromedial tibial tubercle transfer works well also in properly selected patients over the age of 50. The Gigante article provides a logical and effective alternative to PF joint replacement in the treatment of many patients.
The authors have shown clearly that unloading the joint is an important adjunct to resurfacing. Depending on lesion location and extent, I will point out that unloading alone is sometimes all that is needed. Isolated distal and/or lateral facet lesions of the patella can be effectively treated by anteromedial tibial tubercle transfer alone, but we have found, as has the Gigante group, that resurfacing alone is not as successful in such patients.

The Gigante article further reinforces observations and studies of Farr, Minas, Peterson, McKeon, Pidoriano, Carofino, Cole and others, continuing the message that the patellofemoral joint is particularly well suited to preservation, given a logical and careful analysis of mechanical and articular sources of pain and instability, followed by precise surgery and early motion. 